IFT/00-36

hep-ph/0101111

\title{
Phenomenology of the Chargino and Neutralino Systems *
}

\author{
J. KALINOWSKI ${ }^{\dagger}$ \\ Instytut Fizyki TeORETyCZneJ, UniWERSytet WARSZAWSKi \\ Hoża 69, 00681 WarsaW, Poland
}

\begin{abstract}
The chargino and neutralino pair production processes at $e^{+} e^{-}$collisions are explored to determine the underlying SUSY parameters. The sum rules for the couplings are used to check the closure of two-chargino and four-neutralino systems in the minimal supersymmetric extension of the standard model.
\end{abstract}

*) Talk given at the 5th International Linear Collider Workshop (LCWS 2000), Fermilab, Batavia, Illinois, Oct. 24-28, 2000

†) email: kalino@fuw.edu.pl 


\title{
Phenomenology of the Chargino and Neutralino Systems
}

\author{
Jan Kalinowski* \\ ${ }^{*}$ Instytut Fizyki Teoretycznej, Uniwersytet Warszawski \\ Hoża 69, 00681 Warsaw, Poland \\ E-mail: kalino@fuw.edu.pl
}

\begin{abstract}
The chargino and neutralino pair production processes at $e^{+} e^{-}$collisions are explored to determine the underlying SUSY parameters. The sum rules for the couplings are used to check the closure of two-chargino and four-neutralino systems in the minimal supersymmetric extension of the standard model.
\end{abstract}

Assuming that supersymmetry is realized in Nature [1], its confusing signals can be disentangled by combining LHC and LC data. The unique environment of $e^{+} e^{-}$ collisions, with tunable incoming energy and polarized beams in particular [2], will provide means to "measure" the fundamental SUSY parameters independently of theoretical assumptions. This will allow us to confront them with relations following from e.g. grand unification theories [3].

In many SUSY scenarios charginos $\left(\tilde{\chi}_{i}^{ \pm}\right)$and neutralinos $\left(\tilde{\chi}_{i}^{0}\right)$ are among the lightest supersymmetric particles. In the minimal model (MSSM) the chargino sector depends only on the $\mathrm{SU}(2)$ gaugino mass $M_{2}$ (which can be chosen real and positive), the higgsino mass parameter $\mu=|\mu| e^{i \phi_{\mu}}$, and the ratio $\tan \beta\left(=v_{2} / v_{1}\right)$ of the vacuum expectation values of the two neutral Higgs fields, while neutralino sector depends in addition on the $\mathrm{U}(1)$ gaugino mass $M_{1}=\left|M_{1}\right| e^{i \phi_{1}}$. Therefore the chargino and neutralino production processes $e^{+} e^{-} \rightarrow \tilde{\chi}_{i}^{-} \tilde{\chi}_{j}^{+}, \tilde{\chi}_{i}^{0} \tilde{\chi}_{j}^{0}$ may serve as a good starting point towards a systematic and model-independent determination of the fundamental SUSY parameters [4]- [7]. This is conveniently done in two steps first by going from the measurable quantities to physical masses and mixing angles of charginos and neutralinos and then to the fundamental MSSM parameters: $M_{1}$, $M_{2}, \mu$ and $\tan \beta$. For an alternative method of using a global fit, see e.g. [8].

The chargino mass matrix is diagonalized by two different $2 \times 2$ unitary mixing matrices $U_{L}$ and $U_{R}$ acting on left- and right-chiral components of $\tilde{W}^{-}, \tilde{H}^{-}$. They are parameterized by two mixing angles $\phi_{L}$ and $\phi_{R}$ and CP-violating phases $\beta_{L, R}$. The neutralino mass matrix is diagonalized by a single $4 \times 4$ unitary matrix $O_{i j}$ which involves six mixing angles $\theta_{i}$ and ten CP-violating phases. All physical observables can be written in terms of physical masses, mixing angles and $\mathrm{CP}$ - 
phases, which in turn are uniquely determined by the fundamental parameters $M_{1}, M_{2}, \mu, \tan \beta$. Note however, that not all arbitrarily chosen masses, mixing angles and CP-phases are consistent with the MSSM [6].

In $e^{+} e^{-}$collisions the production of chargino pairs receives contributions from $s$-channel $\gamma$ and $Z$ exchanges, and $t$-channel $\tilde{\nu}_{e}$ exchange, while neutralino pairs from $s$-channel $Z$ and $t$ - and $u$-channel selectron $\tilde{e}_{L, R}$ exchanges. For both $\tilde{\chi}_{i}^{-} \tilde{\chi}_{j}^{+}$ and $\tilde{\chi}_{i}^{0} \tilde{\chi}_{j}^{0}$ processes the production amplitude

$$
\mathcal{A}\left[e^{+} e^{-} \rightarrow \tilde{\chi}_{i} \tilde{\chi}_{j}\right]=\frac{e^{2}}{s} Q_{\alpha \beta}^{i j}\left[\bar{v}\left(e^{+}\right) \gamma_{\mu} P_{\alpha} u\left(e^{-}\right)\right]\left[\bar{u}\left(\tilde{\chi}_{i}\right) \gamma^{\mu} P_{\beta} v\left(\tilde{\chi}_{j}\right)\right],
$$

after a Fierz transformation of the $t$ - and $u$-channel contributions, is expressed in terms of four bilinear charges, defined by the chiralities $\alpha, \beta=L, R$ of the lepton and chargino/neutralino currents. The corresponding bilinear charges for the $\tilde{\chi}_{i}^{-} \tilde{\chi}_{j}^{+}$ production take the form

$$
\begin{array}{ll}
Q_{R L}^{i j}=\delta_{i j} D_{R}+\alpha_{i j}^{L} F_{R}, & Q_{L L}^{i j}=\delta_{i j} D_{L}+\alpha_{i j}^{L} F_{L}, \\
Q_{R R}^{i j}=\delta_{i j} D_{R}+\alpha_{i j}^{R} F_{R}, & Q_{L R}^{i j}=\delta_{i j}\left(D_{L}+\frac{T_{\tilde{\nu}}}{4 s_{W}^{2}}\right)+\alpha_{i j}^{R}\left(F_{L}-\frac{T_{\tilde{\nu}}}{4 s_{W}^{2}}\right),
\end{array}
$$

while in the case of $\tilde{\chi}_{i}^{0} \tilde{\chi}_{j}^{0}$ production they are

$$
\begin{array}{ll}
Q_{R L}^{i j}=+\frac{D_{Z}}{c_{W}^{2}} \mathcal{Z}_{i j}+T_{\tilde{e}_{R}} g_{R i j}, & Q_{L L}^{i j}=+\frac{D_{Z}}{s_{W}^{2} c_{W}^{2}}\left(s_{W}^{2}-\frac{1}{2}\right) \mathcal{Z}_{i j}-U_{\tilde{e}_{L}} g_{L i j}, \\
Q_{R R}^{i j}=-\frac{D_{Z}}{c_{W}^{Z}} \mathcal{Z}_{i j}^{*}-U_{\tilde{e}_{R}} g_{R i j}^{*}, & Q_{L R}^{i j}=-\frac{D_{Z}}{s_{W}^{2} c_{W}^{2}}\left(s_{W}^{2}-\frac{1}{2}\right) \mathcal{Z}_{i j}^{*}+T_{\tilde{e}_{L}} g_{L i j}^{*},
\end{array}
$$

with $s^{-}, t-$, and $u$-channel propagators $D_{L}=1+\frac{D_{Z}}{s_{W}^{2} c_{W}^{2}}\left(s_{W}^{2}-\frac{1}{2}\right)\left(s_{W}^{2}-\frac{3}{4}\right), F_{L}=$ $\frac{D_{Z}}{4 s_{W}^{2} c_{W}^{2}}\left(s_{W}^{2}-\frac{1}{2}\right), D_{R}=1+\frac{D_{Z}}{c_{W}^{2}}\left(s_{W}^{2}-\frac{3}{4}\right), F_{R}=\frac{D_{Z}}{4 c_{W}^{2}}, D_{Z}=s /\left(s-m_{Z}^{2}+i m_{Z} \Gamma_{Z}\right)$, $T_{a}=s /\left(t-m_{a}^{2}\right), U_{a}=s /\left(u-m_{a}^{2}\right)$. The couplings $\alpha_{i j}^{L, R}=\delta_{i j}(-1)^{i} \cos 2 \phi_{L, R}+$ $\left(1-\delta_{i j}\right) \sin 2 \phi_{L, R} e^{-i \beta_{L, R}}, g_{R i j}=\frac{1}{c_{W}^{2}} O_{i 1} O_{j 1}^{*}, g_{L i j}=\frac{1}{4 s_{W}^{2} c_{W}^{2}}\left(O_{i 2} c_{W}+O_{i 1} s_{W}\right)\left(O_{j 2}^{*} c_{W}+\right.$ $\left.O_{j 1}^{*} s_{W}\right)$ and $\mathcal{Z}_{i j}=\frac{1}{2}\left(O_{i 3} O_{j 3}^{*}-O_{i 4} O_{j 4}^{*}\right)$ are written in terms of the mixing angles $\phi_{L, R}$ and CP-phases $\beta_{L, R}$ (for charginos), and the diagonalization matrix elements $O_{i j}$ (for neutralinos). Note that for the chargino case the $Q_{\alpha \beta}^{i j}$ are linear in $s_{2 L, R} \equiv$ $\sin 2 \phi_{L, R}$ and $c_{2 L, R} \equiv \cos 2 \phi_{L, R}$, and the phases $\beta_{L, R}$ will disappear in quantities not sensitive to chargino helicities. The $\tilde{\nu}_{e}$ exchange contributes only to the $L R$ chargino amplitude. In contrast, in the neutralino case the $Q_{\alpha \beta}^{i j}$ are quadratic in $O_{i j}$ and selectron exchanges contribute to all neutralino amplitudes.

We define the polar angle $\Theta$ and azimuthal angle $\Phi$ of the produced $\tilde{\chi}_{i}$ in the reference frame given by the $e^{-}$momentum direction as the $z$-axis and the $e^{-}$ transverse polarization vector as the $x$-axis. The polarized differential cross section $\mathrm{d} \sigma^{i j} / \mathrm{d} \Omega\left[e^{+} e^{-} \rightarrow \tilde{\chi}_{i} \tilde{\chi}_{j}\right]$, for the $e^{-}$and $e^{+}$polarization vectors $P=\left(P_{T}, 0, P_{L}\right)$ and $\bar{P}=\left(\bar{P}_{T} \cos \eta, \bar{P}_{T} \sin \eta,-\bar{P}_{L}\right)$ respectively, is given by

$$
\frac{\mathrm{d} \sigma^{i j}}{\mathrm{~d} \Omega}=\frac{\alpha^{2}}{16 s} \lambda^{1 / 2}\left[\left(1-P_{L} \bar{P}_{L}\right) \sum_{\mathrm{unp}}^{i j}+\left(P_{L}-\bar{P}_{L}\right) \Sigma_{L L}^{i j}+P_{T} \bar{P}_{T} \cos (2 \Phi-\eta) \Sigma_{T T}^{i j}\right],
$$




$$
\begin{aligned}
\Sigma_{\mathrm{unp}}^{i j} & =4\left\{\left[1-\left(\mu_{i}^{2}-\mu_{j}^{2}\right)^{2}+\lambda \cos ^{2} \Theta\right] Q_{1}+4 \mu_{i} \mu_{j} Q_{2}+2 \lambda^{1 / 2} Q_{3} \cos \Theta\right\}, \\
\Sigma_{L L}^{i j} & =4\left\{\left[1-\left(\mu_{i}^{2}-\mu_{j}^{2}\right)^{2}+\lambda \cos ^{2} \Theta\right] Q_{1}^{\prime}+4 \mu_{i} \mu_{j} Q_{2}^{\prime}+2 \lambda^{1 / 2} Q_{3}^{\prime} \cos \Theta\right\}, \\
\Sigma_{T T}^{i j} & =-4 \lambda \sin ^{2} \Theta Q_{5},
\end{aligned}
$$

where $\lambda=\left(1-\left(\mu_{i}+\mu_{j}\right)^{2}\right)\left(1-\left(\mu_{i}-\mu_{j}\right)^{2}\right)$ is the two-body phase space function, $\mu_{i}^{2}=m_{\tilde{\chi}_{i}}^{2} / s$. The quartic charges are

$$
\begin{array}{ll}
Q_{1}=\frac{1}{4}\left[\left|Q_{R R}\right|^{2}+\left|Q_{L L}\right|^{2}+\left|Q_{R L}\right|^{2}+\left|Q_{L R}\right|^{2}\right], & Q_{2}=\frac{1}{2} \operatorname{Re}\left[Q_{R R} Q_{R L}^{*}+Q_{L L} Q_{L R}^{*}\right], \\
Q_{3}=\frac{1}{4}\left[\left|Q_{R R}\right|^{2}+\left|Q_{L L}\right|^{2}-\left|Q_{R L}\right|^{2}-\left|Q_{L R}\right|^{2}\right], & Q_{5}=\frac{1}{2} \operatorname{Re}\left[Q_{L R} Q_{R R}^{*}+Q_{L L} Q_{R L}^{*}\right], \\
Q_{1}^{\prime}=\frac{1}{4}\left[\left|Q_{R R}\right|^{2}+\left|Q_{R L}\right|^{2}-\left|Q_{L R}\right|^{2}-\left|Q_{L L}\right|^{2}\right], & Q_{2}^{\prime}=\frac{1}{2} \operatorname{Re}\left[Q_{R R} Q_{R L}^{*}-Q_{L L} Q_{L R}^{*}\right], \\
Q_{3}^{\prime}=\frac{1}{4}\left[\left|Q_{R R}\right|^{2}+\left|Q_{L R}\right|^{2}-\left|Q_{R L}\right|^{2}-\left|Q_{L L}\right|^{2}\right] . &
\end{array}
$$

The charges $Q_{1}$ to $Q_{5}$ are manifestly $\mathrm{P}$-even, while $Q_{1}^{\prime}, Q_{2}^{\prime}$ and $Q_{3}^{\prime}$ are $\mathrm{P}$-odd.

Since charginos and heavier neutralinos decay mainly into the invisible lightest neutralinos and SM fermion pairs, the production angles $\Theta$ and $\Phi$ cannot be determined completely on an event-by-event basis. Integrating over $\Theta$ and $\Phi$, the dependence of the total production cross sections on beam polarization can be exploited to extract information on the mixing states of charginos/neutralinos and on masses of exchanged sneutrinos/selectrons [7]. For the purpose of determining fundamental parameters it is enough to consider the following integrated polarization-dependent cross sections as physical observables [5]:

$$
\sigma_{R, L}^{i j}=\int \mathrm{d} \Omega \frac{\mathrm{d} \sigma^{i j}}{\mathrm{~d} \Omega}\left[P_{L}= \pm P^{\max }, \bar{P}_{L}=\mp \bar{P}^{\max }\right]
$$

where $P^{\max }$ and $\bar{P}^{\max }$ are the maximum longitudinal polarizations of $e^{-}$and $e^{+}$.

The two-state mixing of charginos and four-state mixing of neutralinos lead to sum rules for the chargino and for the neutralino couplings. They can be formulated in terms of the squares of the bilinear charges. This follows from the observation that the mixing matrices are unitary. For example, the following general sum rules can be derived for the two-state chargino system at tree level:

$$
\begin{aligned}
& \sum_{i, j=1,2}\left|Q_{\alpha \beta}^{i j}\right|^{2}=2\left(\left|D_{\alpha}\right|^{2}+\left|F_{\alpha}\right|^{2}\right), \quad(\alpha \beta)=(L L, R L, R R), \\
& \sum_{i, j=1,2}\left|Q_{L R}^{i j}\right|^{2}=2\left(\left|D_{L}+\frac{T_{\tilde{\nu}}}{4 s_{W}^{2}}\right|^{2}+\left|F_{L}-\frac{T_{\tilde{\nu}}}{4 s_{W}^{2}}\right|^{2}\right) .
\end{aligned}
$$

The right-hand side of (8) is independent of any supersymmetric parameters, while (9) involves the sneutrino mass. Similarly, the corresponding sum rules for the neutralino case can be derived. The validity of these sum rules is reflected in both the quartic charges and the production cross sections although, due to mass effects and the $t / u$-channel sfermion exchanges, they are more involved. Only asymptotically at high energies the sum rules (like in eqs.8,9) can be transformed directly into the sum rules for the total cross sections. We find [9] 


$$
\Sigma_{i j=1,2} \sigma\left(\tilde{\chi}_{i}^{-} \tilde{\chi}_{j}^{+}\right)=\frac{347 \mathrm{fb}^{-1}}{s / \mathrm{TeV}^{2}}, \quad \Sigma_{i j=1, . ., 4} \sigma\left(\tilde{\chi}_{i}^{0} \tilde{\chi}_{j}^{0}\right)=\frac{323 \mathrm{fb}^{-1}}{s / \mathrm{TeV}^{2}}
$$

Nevertheless, the fact that all the physical observables are functions of elements of the mixing matrices enables us to relate the cross sections with the mixing angles. This has been worked out explicitly for the chargino case. The six polarized cross sections $\sigma_{L, R}^{i j}$ can be written as linear combinations of six formally independent variables $\left\{z_{k}\right\}=\left\{1, c_{2 L}, c_{2 R}, c_{2 L}^{2}, c_{2 R}^{2}, c_{2 L} c_{2 R}\right\}$, with coefficients which are known functions of chargino masses, sneutrino mass (taken from e.g. sneutrino pair production) and other known parameters (the scattering energy, SM couplings etc.). Inverting, the variables $z_{i}$ are given in terms of the observables $\sigma_{L, R}^{i j}$. Since $z_{i}$ are not independent, viz. $z_{4}=z_{2}^{2}, z_{5}=z_{3}^{2}, z_{6}=z_{2} z_{3}$, we obtain several non-trivial relations among the observables in the chargino sector. The failure of saturating any of these sum rules by the measured cross sections would signal that the chargino two-state $\left\{\tilde{\chi}_{1}^{ \pm}, \tilde{\chi}_{2}^{ \pm}\right\}$system is not complete and additional states mix in. If the sum rules are satisfied, the $z_{2}$ and $z_{3}$ are the required mixing parameters $\cos 2 \phi_{L}$ and $\cos 2 \phi_{R}$. Similar techniques can be worked out for the neutralino system [9].

It has recently been demonstrated [5] that if the collider energy is sufficient to produce the light and heavy chargino states in pairs, the underlying fundamental SUSY parameters, $M_{2},|\mu|, \cos \phi_{\mu}$ and $\tan \beta$, can be extracted unambiguously from chargino masses and production cross sections with polarized electron beams. The chargino masses can be determined very precisely from the sharp rise of the cross sections. Defining $\Delta=m_{\tilde{\chi}_{2}^{ \pm}}^{2}-m_{\tilde{\chi}_{1}^{ \pm}}^{2}$, the fundamental parameters are

$$
\begin{aligned}
& \tan \beta=\left[\left(4 m_{W}^{2}+\Delta\left(\cos 2 \phi_{R}-\cos 2 \phi_{L}\right)\right) /\left(4 m_{W}^{2}-\Delta\left(\cos 2 \phi_{R}-\cos 2 \phi_{L}\right)\right)\right]^{1 / 2}, \\
& M_{2}=\frac{1}{2}\left[2\left(m_{\tilde{\chi}_{2}^{ \pm}}^{2}+m_{\tilde{\chi}_{1}^{ \pm}}^{2}-2 m_{W}^{2}\right)-\Delta\left(\cos 2 \phi_{R}+\cos 2 \phi_{L}\right)\right]^{1 / 2}, \\
& |\mu|=\frac{1}{2}\left[2\left(m_{\tilde{\chi}_{2}^{ \pm}}^{2}+m_{\tilde{\chi}_{1}^{ \pm}}^{2}-2 m_{W}^{2}\right)+\Delta\left(\cos 2 \phi_{R}+\cos 2 \phi_{L}\right)\right]^{1 / 2}, \\
& B=\left[\Delta^{2}-\left(M_{2}^{2}-\mu^{2}\right)^{2}-4 m_{W}^{2}\left(M_{2}^{2}+\mu^{2}+m_{W}^{2} \cos ^{2} 2 \beta\right)\right] / 8 m_{W}^{2} M_{2}|\mu| \sin 2 \beta,
\end{aligned}
$$

where $B=\operatorname{sign}(\mu)$ in $\mathrm{CP}$-invariant and $B=\cos \phi_{\mu}$ in $\mathrm{CP}$-noninvariant theories.

With the additional knowledge of the lightest neutralino mass, $M_{1}$ can be determined up to a two-fold ambiguity. This ambiguity can be resolved e.g. by measuring neutralino production cross sections.

As an example we consider a CP-invariant MSUGRA point with $\tan \beta=3$, $m_{0}=100 \mathrm{GeV}, M_{1 / 2}=200 \mathrm{GeV}, \operatorname{sign}(\mu)>0$. Assuming $1 \sigma$ statistical errors for cross section measurements at $\sqrt{s}=800 \mathrm{GeV}$ and $500 \mathrm{fb}^{-1}$ per left- and rightpolarized electron beams, and the mass of $\tilde{\chi}_{1}^{ \pm}$to be measured with an accuracy of $50 \mathrm{MeV}$ and masses of $\tilde{\chi}_{2}^{ \pm}$and $\tilde{\chi}_{1}^{0}$ with $200 \mathrm{MeV}$, the following accuracies may be expected: $M_{2}=152 \pm 1.8 \mathrm{GeV}, \mu=316 \pm 0.9 \mathrm{GeV}, \tan \beta=3 \pm 0.7$ and $M_{1}=78 \pm 0.7 \mathrm{GeV}$. Slightly better errors are obtained for a high $\tan \beta=30$ scenario, except for the $\tan \beta$ parameter which this time is poorly determined as $\tan \beta>20$. 
In determining the above error of $\tan \beta$ the information that $\left|\cos \phi_{\mu}\right|=1$ (i.e. no $\mathrm{CP}$-violating phase $\phi_{\mu}$ ) has been used [10]. If the value of $\tan \beta$ can be measured more precisely elsewhere (e.g. in the Higgs sector with $\sim 10 \%$ error), then eq.(13) can be used to verify that $\cos \phi_{\mu}=1 \pm 0.1$.

To summarize, the chargino and neutralino sectors can be analyzed independently of each other. This is important since the structure of the neutralino sector may potentially be very complex in theories beyond the MSSM. The sum rules for the production cross sections provide a consistency check of the underlying theoretical picture of the chargino and neutralino systems and the experimental procedure in extracting the fundamental parameters. The discussion presented here has been carried out at the tree level; the higher-order corrections [11] (which include parameters from other sectors of the MSSM) demand iterative expansions in global analyses at the very end.

Acknowledgments: Invaluable discussions with my collaborators are acknowledged. This work has been supported by the KBN Grant 2 P03B 06018.

\section{REFERENCES}

1. H. Murayama, these proceedings.

2. G. Moortgat-Pick, these proceedings.

3. G.A. Blair, W. Porod and P.M. Zerwas, Phys. Rev. D63 (2001) 017703.

4. T. Tsukamoto et al., Phys. Rev. D51 (1995) 3153; J.L. Feng et al., Phys. Rev. D52 (1995) 1418.

5. S.Y. Choi et al., Eur. Phys. J. C7 (1999) 123, Eur. Phys. J. C8 (1999) 669, Eur. Phys. J. C14 (2000) 535, Phys. Lett. B479 (2000) 235, Phys. Rev. D61 (2000) 075004 .

6. J.L. Kneur and G. Moultaka, Phys. Rev. D59 (1999) 015005, Phys. Rev. D61 (2000) 075004.

7. G. Moortgat-Pick et al., Eur. Phys. J. C7 (1999) 113, Eur. Phys. J. C9 (1999) 521, Phys. Rev. D59 (1999) 015016, hep-ph/0002253, hep-ph/000481, hep-ph/0007222.

8. V. Barger et al., Phys. Lett. B475 (2000) 342.

9. S.Y. Choi, J. Kalinowski, G. Moortgat-Pick and P.M. Zerwas, in preparation.

10. S.Y. Choi and J. Kalinowski, hep-ph/9910431; J. Kalinowski, Pramana 55 (2000) 259.

11. M.A. Diaz, S.F. King and D.A. Ross, Nucl. Phys. B529 (1998) 23, hep-ph/0008117, M.A. Diaz, these proceedings; T. Blank and W. Hollik, hep-ph/0011092. 